\title{
Columna Falsa De Cargadores De Celulares Libre De Baldosas Piezoeléctrica Para La Biblioteca De La Universidad Nacional Tecnológica De Lima Sur
}

Anwar Yarín, Jorge Sanchez dol , Edgard Hernandez, Kevin Rodríguez, Estudiante Patrick Abanto

Universidad Nacional Tecnológica de Lima Sur, Lima, Perú

Recibido: $15 / 11 / 2020 \quad$ Revisado: 21/11/2020 Aceptado: 26/11/2020 Publicado: 28/01/2021

\section{Resumen:}

Para poder abasto de energía eléctrica a un sector estudiantil que demanda más puntos de salida y para su comodidad, se indaga en la aplicación de obtención de energía eléctrica por medio de baldosas piezoeléctricas que presenta cerámicos piezoeléctricos que al deformarse por un movimiento mecánicos producen energía eléctrico, es por ello que se analiza los posibles lugares de la UNTELS para la aplicación de esta tecnología, según un dato por encuesta los estudiantes en general prefieren la biblioteca como zona de estar en el cual se produce una cantidad considerable de personas que generan suficiente energía para abastecer el sistema piezoeléctrico. Los estudiantes son quienes avanzan por estos puntos de la universidad y pueden dar fe de su punto estratégico.

Palabras clave: Piezoelectricidad, Energía, Libre, cargador, usuarios.

\begin{abstract}
:
In order to supply electrical energy to a student sector that demands more exit points and for their comfort, the application of obtaining electrical energy by means of piezoelectric tiles that presents piezoelectric ceramics is investigated that when deformed by mechanical movement produce electrical energy, that is why the possible places of the UNTELS for the application of this technology are analyzed, according to a survey data, students in general prefer the library as a living area in which a considerable number of people are produced that generate enough energy to supply the piezoelectric system. Students are the ones who advance through these points of the university and can attest to their strategic point.
\end{abstract}

Keywords: Piezoelectricity, Energy, Free, charger, users. 


\section{Introducción:}

En el momento que se desea realizar un estudio en la universidad siempre hay cierta dependencia a medios electrónicos, ya sea para comunicación o búsqueda de información o realización de documentos importantes, el gasto de energía es inevitable, sumamos a ello el hecho de no contar muchos puntos de recarga de celulares, esto genera un problema en la comunidad Untelsina, es así que se investiga al problema de falta de lugares de conexión para celulares que cumplan la función de recargar los celulares. Es por ello que se plantea realizar un sistema adecuado para la recolección de energía libre que sea capaz de abastecer los cargadores y equipos electrónicos que irán en él. La solución hipotética es la aplicación de energía piezoeléctrica, una energía libre de contaminación y autónomo que dependerá de los mismos usuarios cargar todo el sistema, es así que los mismos usuarios abastecen a sus equipos electrónicos. La idea en cuestión es emplearla en un lugar estratégico de la UNTELS para que los beneficiarios sean la comunidad Untelsina. Por ello que mediante la Línea de electrónica en sub-línea de energía renovables se da la idea de implementar un sistema piezoeléctrico que puede autoabastecerse y de energía gratis a la comunidad Untelsina.

\section{Metodología:}

La investigación consiste en el diseño final de un prototipo de sistema eléctrico capaz de auto sostenerse mediante la recolección energética de las pisadas. Este fenómeno es conocido como piezoelectricidad que ocurre al choque de cristales finos en una masa piezoeléctrica, este choque genera traslación de los electrones hacia los cables que trasladan la electricidad hacia un rectificador de corriente para no tener una corriente alterna y se pueda emplear para la alimentación de equipos electrónicos como en este caso. Luego del rectificador la corriente viaja a un condensador que termina de regularizar la corriente y conducirla hacia una batería, esta a su vez alimenta un equipo electrónico, en este los equipos electrónicos como celulares o laptops.

Esta investigación es un estudio Cuantitativo de la producción energética, consumo al día y el número de personas en circulación en cierta área del transporte Público. 
De estos variables básicas se determina:

Variables:

Variables independientes (X):

El material, sistema piezoeléctrico y el estudio de un sector de transporte público con personas.

Variables dependientes (Y):

Humedad, climáticos, aditamentos adicionales, datos originados por las variables independientes.

Tabla 1: Las variables y su clasificación

\begin{tabular}{|c|c|c|c|}
\hline \multicolumn{4}{|c|}{ Piezoelectricidad } \\
\hline Variable & Indicadores de medición & Índice, valores o & Instrumento de \\
\hline \multirow{7}{*}{$=\cdots$} & Duración del material piezoeléctrico & $28-30$ dias & Pluviómetro \\
\hline & $\begin{array}{l}\text { Mantenimiento del sistema } \\
\text { piezoeléctrico }\end{array}$ & 3 meses & Higrómetro \\
\hline & $\begin{array}{lll}\text { Resistencia } & \text { del } & \text { material } \\
\text { piezoeléctrico } & & \end{array}$ & $5 \times 10^{7} \mathrm{~N}$ & Paloteo \\
\hline & Vida útil & 2.5 años & \\
\hline & Cantidad de Resortes & $10 \mathrm{xm}^{2}$ & \\
\hline & Cantidad de material piezoeléctrico & $333 \mathrm{xm}^{2}$ & \\
\hline & Tamaño del material piezoeléctrico & $15 \times 0.9 \mathrm{~mm}$ & Calibrador \\
\hline \multirow{7}{*}{$\begin{array}{c}\text { Variables } \\
\text { dependientes }\end{array}$} & Lluvia & $7,74 \mathrm{~mm}$ & Pluviómetro \\
\hline & \multirow[b]{2}{*}{ Altura de los pasos de las personas } & Hombres $0.415 \mathrm{~m}$ & \\
\hline & & Mujeres $0.413 \mathrm{~m}$ & \\
\hline & Humedad & $85,77 \%$ & Higrómetro \\
\hline & Deformación de resorte & $0.003 \mathrm{~m}$ & \\
\hline & Presión por pisada & $0.486 \mathrm{~N}$ & \\
\hline & Cantidad de personas & $\begin{array}{c}562 \text { personas } \\
\text { /Hora }\end{array}$ & Paloteo \\
\hline
\end{tabular}


Diseño de la investigación:

El diseño presentado es experimental.

Población y muestra:

Población:

La comunidad UNTELSINA de Villa el Salvador. Gracias a que el proyecto se establecerá ahí. Muestra:

El grupo de personas que pasan a través de la puerta principal de la universidad puesto a su gran número de personas que afluyen ahí, ser de ingreso peatonal y ser adecuado para el prototipo en este proyecto; sin embargo, la aplicabilidad puede extender a diversos puntos de la universidad.

Técnicas e instrumentos de recolección de datos:

Paloteo: Es estudio o método estadístico que satisface la necesidad que se origina cuando existe un gran volumen de información y que puede ser utilizado para organizar, presentar, analizar e interpretar la información efectivamente. De este modo, mediante su aplicación puedes extraer conclusiones válidas y tomar decisiones razonables respecto al hecho, universo o población que se estudia.

Para este proyecto se utilizó esta técnica el cual nos indica el número de personas que transitan a diario mediante la observación y conteo por la puerta principal de nuestra casa de estudio de esta manera se logró obtener información fiable de frecuencia en la cual se posibilita la mayor generación de energía a almacenar

BIOTECH \& ENGINEERING Untels. Ene - jun.1(1), 2021; ISSN: 2788-4295; 82 - 99 


\section{Contribuciones:}

\section{Marco teórico:}

\section{Piezoelectricidad:}

La piezoelectricidad es un fenómeno que ocurre en determinados cristales que, al ser sometidos a una carga o presión, se genera un diferencial de potencial eléctrico en el cual la polaridad depende del sentido en que se aplique la fuerza.

Para que en la materia ocurra la propiedad de la piezoelectricidad debe cristalizar en sistemas que carezcan de centro de simetría y por la tanto de eje polar. Si se ejerce presión en los extremos del eje polar se produce polarización, en otros términos, es el flujo de electrones que se dirigen a un extremo y genera en él una carga negativa mientras en el extremo opuesto se genera una carga positiva.

\section{Materiales Piezoeléctricos:}

Los materiales piezoeléctricos naturales son: el cuarzo, la turmalina, sal de Dochelle, etc. Si bien es cierto que podemos usar estos materiales para causar electricidad mediante la presión, pero no es usada directamente porque no producen tanta energía como los piezoeléctricos sintéticos que están compuestos por: cristales de niobato de litio (LiNbO3), cuarzo sintético, titanio circonato de plomo (PZT), polifluoruro de vinilideno (PVDF), etc.

Entre las características de los piezoeléctricos sintéticos se puede mencionar a su relativa insensibilidad a la temperatura, factores elevados de conversión de energía eléctrica y energía mecánica, entre otros atributos, hacen que a estos materiales se les de gran uso.

Este proceso también ocurre con el proceso inverso, al aplicarse una corriente eléctrica sobre el material esto produce una deformación, pero al dejar de aplicar dicha corriente volverá a su estado natural. 


\title{
Estudio de locación:
}

\author{
Demanda objetiva
}

Como sabemos el proyecto consiste en implementar un grupo de baldosas en lugares altamente transitados con la finalidad que nos entregue la mayor cantidad de energía, por lo cual mencionaremos los lugares posibles para la instalación de estos pisos y las variables que tenemos que tener en cuenta que son el clima y la transitividad.

Entrada de la universidad Nacional Tecnológica de Lima sur

El acceso a la universidad es un lugar por donde circulan alrededor de 2640 personas, incluyendo alumnos, docentes, administrativos y empleados. Lo cual nos daría una excelente cantidad de energía, pero por contraparte el piezoeléctrico no puede estar expuesto al contacto con el agua. Tomando en cuenta el clima de lima, Perú. Tenemos que subrayar este punto negativo ya que el piezo eléctrico no puede estar en contacto con la lluvia, sabiendo que en la entrada de la universidad no poseemos ningún tipo de techo que nos proteja de esta.

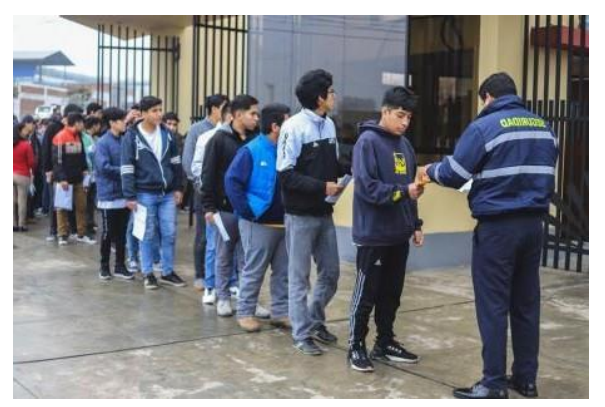

Figura 1: Entrada de la Universidad Nacional Tecnológica de Lima Sur.

Cafetería de la universidad Nacional Tecnológica de Lima sur

El área de la cafetería también es un espacio donde habitualmente se reúne la comunidad estudiantil ya sea para ingerir algún alimento, bebida o golosina.

Lamentablemente este lugar no es tan visitado regularmente y el área que esta posee no es la más grande posible siendo solo de $50 \mathrm{~m} 2$ por esto podemos decir que el proyecto no sería tan rentable BIOTECH \& ENGINEERING Untels. Ene - jun.1(1), 2021; ISSN: 2788 - 4295; 82 - 99 
en este lugar, pero como dato positivo es que no tendríamos ningún inconveniente con el tema del clima ya que este es un lugar cerrado.

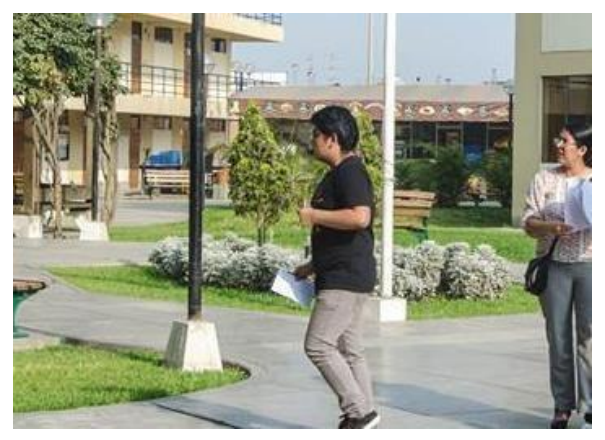

Figura 2: Cafetín de la Universidad Nacional Tecnológica de Lima Sur:

Las escaleras de los edificios de la universidad Nacional Tecnológica de Lima sur:

Al colocar las baldosas en las escaleras se puede reunir gran cantidad de energía ya que, el efecto piezoeléctrico generado por la presión de las personas al caminar es mayor, debido a que al bajar por una escalera aumenta la fuerza con la que el material piso eléctrico es presionado generando así más energía eléctrica.

Sumado a la alta transitividad y a la protección parcial que ejerce los techos de estos edificios podríamos concluir que este lugar es un gran candidato. 


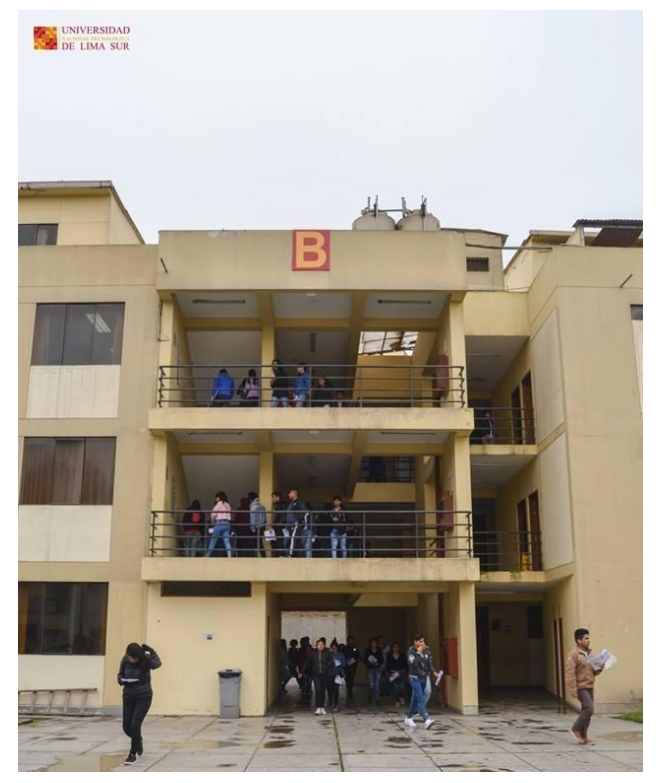

Figura 3: Escaleras de la Universidad Nacional Tecnológica de Lima Sur:

Biblioteca Santiago Antúnez de Mayolo:

Esta biblioteca se encuentra dentro de la Universidad Nacional Tecnológica de Lima Sur tiene aproximadamente 300 visitantes diarios, un área de cerca de $250 \mathrm{~m} 2$ y posee 2 niveles.

El tránsito en esta biblioteca es constante ya sea por la entrada de alumnos o jóvenes del distrito en el que se ubica. Con respecto a la exposición con líquidos es casi mínima ya que prohíben el ingreso con bebidas y alimentos a dicho lugar. Siendo este el lugar elegido por nosotros por las características mencionadas y ya que podemos solucionar la problemática de falta de tomacorrientes que existe en la biblioteca por la alta demanda de conectar equipos electrónicos. 


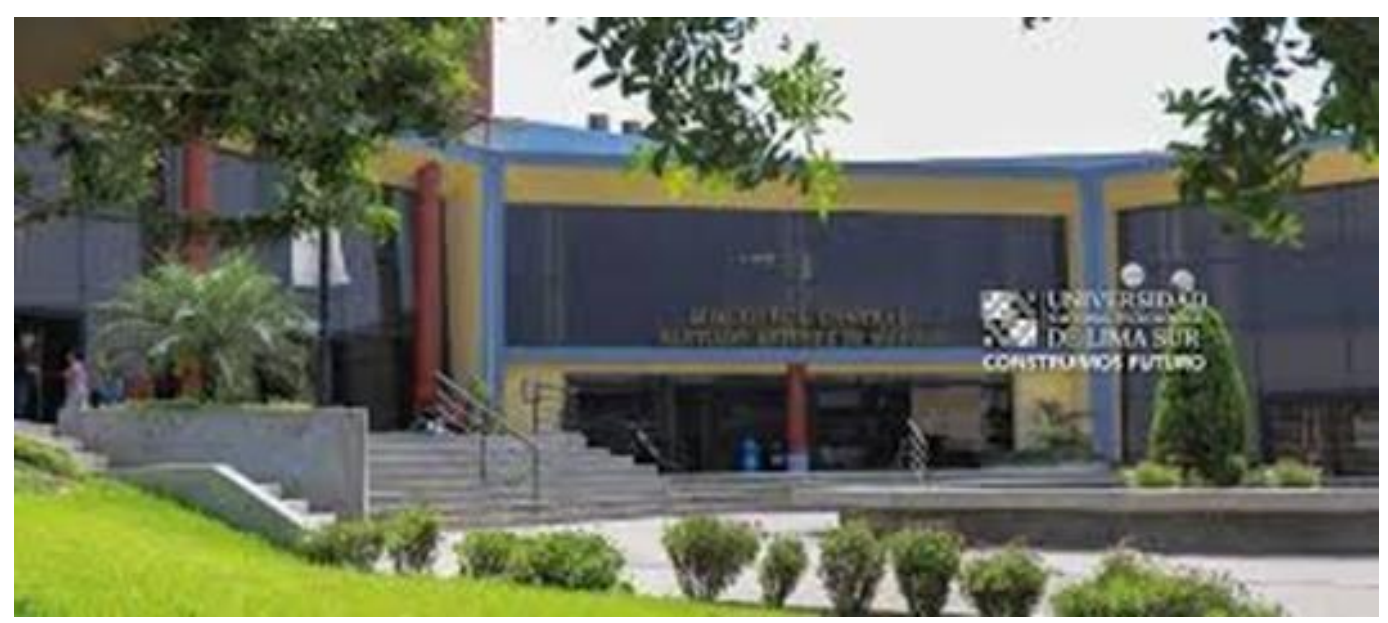

Figura 4: Biblioteca Santiago Antúnez de Mayolo

\section{Diseño}

El estado de arte es una pared Falsa con cargadores en cajas con puertas para la colocación de los equipos electrónicos y aguarden adentro, la batería interna se ubica dentro de la pared falsa, así como los dispositivos de rectificación de corriente eléctrica. 


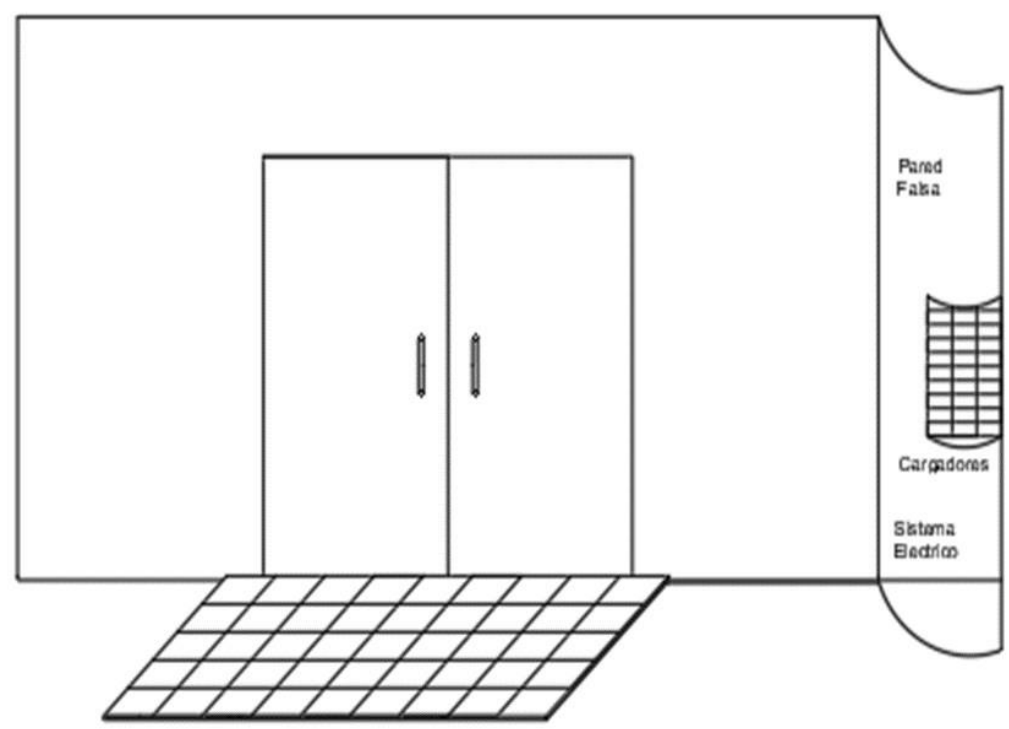

Figura 5: Vista general del sistema de Columna falsa de cargadores de celulares libre

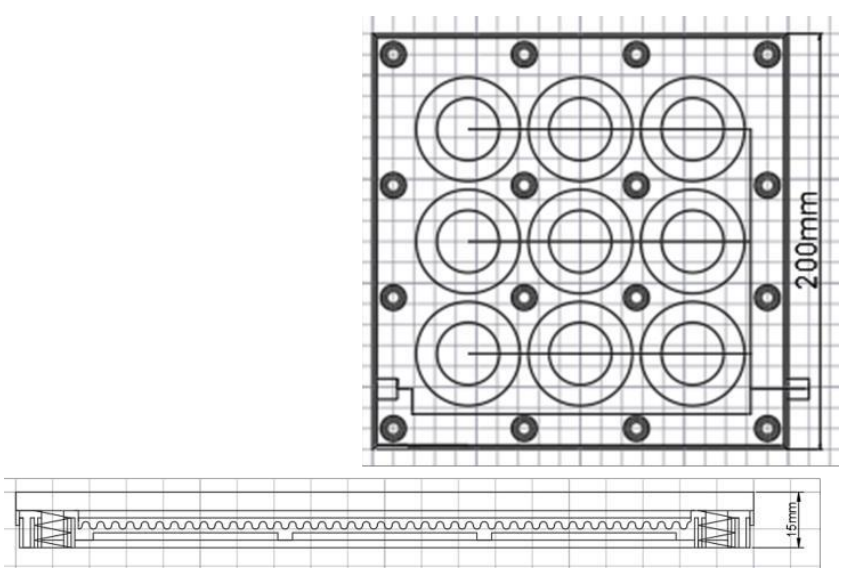

Figura 6: Vista interior del sistema piezoeléctrico de una baldosa

BIOTECH \& ENGINEERING Untels. Ene - jun.1(1), 2021; ISSN: $2788-4295 ; 82-99$ 
La cantidad de consumidores viéndolo del lado de la utilización de la energía en la Universidad Nacional Tecnológica de Lima sur serian algo de 150 personas al día ya que son el numero promedio de estudiantes que cargan sus equipos electrónicos. Y viéndolo por el lado de la venta de nuestro producto, la proyección que tenemos es muy positiva ya que al realizar encuestas a diferentes empresas hemos tenido una buena reacción de estas. La estructura de la encuesta realizada es la siguiente:

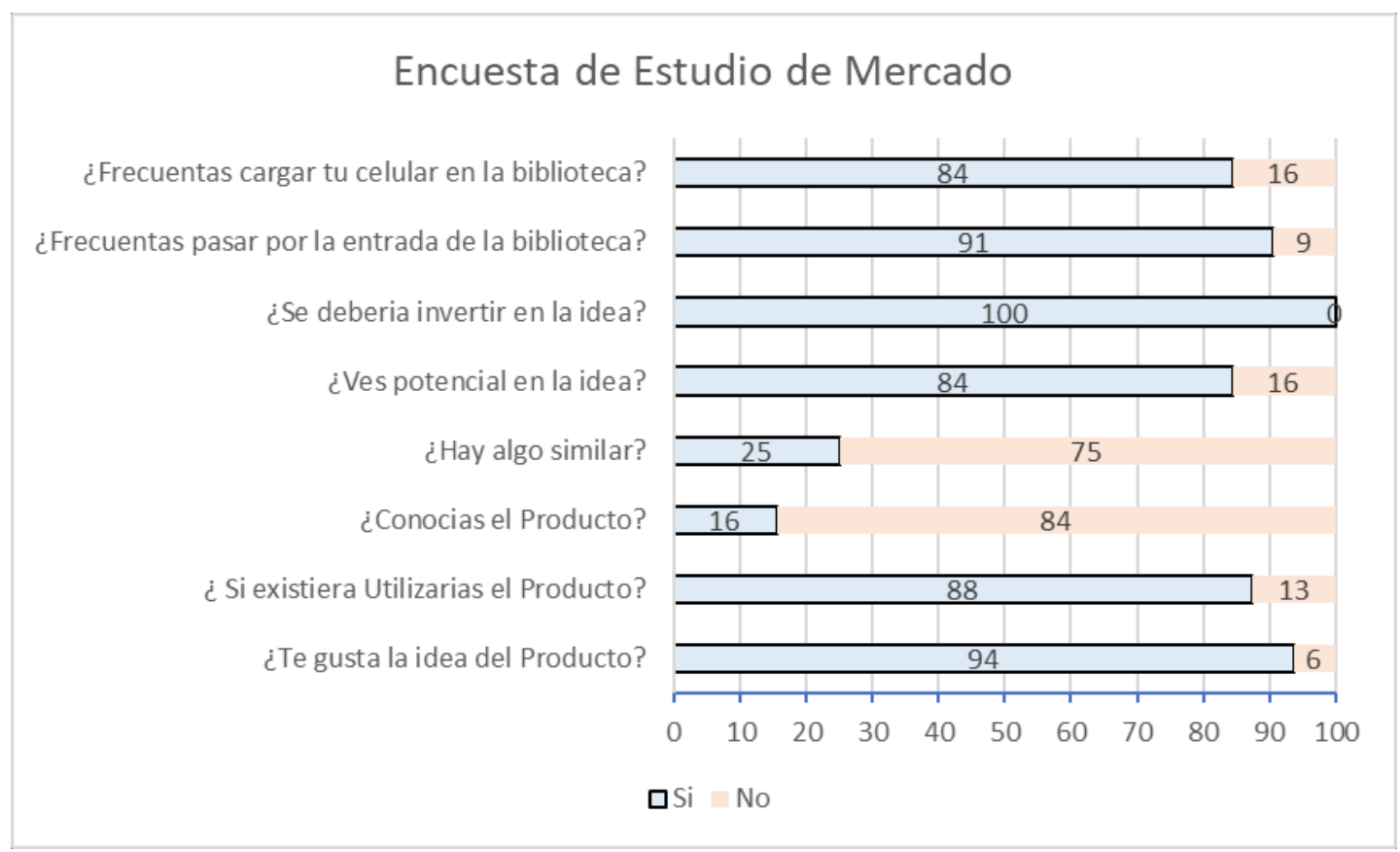

Gráfico 1: Resultado de encuesta estudiantil acerca de la aplicabilidad de las baldosas piezoeléctricas.

\section{Estudio de mercado de los componentes de los diseños de primer prototipo:}

Para la selección del material piezoeléctrico y de red electrónica se hace un estudio para la fabricación de las distintas baldosas en así que en la tabla 2, compuestos de elementos electrónicas y mecánicos complementarios, en la tabla 3observaremos las piezas de canaletas interiores que se encuentran alrededor de las baldosas también, y la tabla 4 que envía data de los elementos superficiales idóneos para la elaboración de superficies exteriores. 


\begin{tabular}{|l|l|l|l|}
\hline \multirow{2}{*}{ RESORTE } & \multirow{4}{*}{} & \multirow{2}{*}{} \\
\cline { 2 - 2 } & TIPOS DE RESORTES & \\
\hline
\end{tabular}

\begin{tabular}{|c|c|c|c|c|c|}
\hline & COMPRESIÓN & $\begin{array}{l}\text { EXTENSI } \\
\text { ÓN }\end{array}$ & TORSIÓN & CONDENSADOR & $\begin{array}{c}\text { ALAMBRAD } \\
\mathbf{O}\end{array}$ \\
\hline $\begin{array}{c}\text { PRECI } \\
\text { O }\end{array}$ & $15-150$ soles & $55-100$ soles & $50-150$ soles & $1-10$ soles & $25-50$ soles \\
\hline $\begin{array}{c}\text { CARAC } \\
\text { TERÍST } \\
\text { ICA }\end{array}$ & $\begin{array}{l}\text { Destinado a } \\
\text { soportar } \\
\text { esfuerzos de } \\
\text { compresión y } \\
\text { choque }\end{array}$ & $\begin{array}{ll}\text { Deben } & \\
\text { presentar } & \text { sus } \\
\text { extremos } & \\
\text { curvados } & \text { en } \\
\text { forma } & \text { de } \\
\text { gancho } & \end{array}$ & $\begin{array}{l}\text { Se deforma al } \\
\text { ser sometido } \\
\text { por sus } \\
\text { extremos a un } \\
\text { par de fuerzas } \\
\text { perpendicular } \\
\text { es a su eje }\end{array}$ & $\begin{array}{l}\text { Está } \\
\text { formado por } \\
\text { un par de } \\
\text { superficies } \\
\text { conductoras } \\
\text {, } \\
\text { generalment } \\
\text { e en forma } \\
\text { de láminas o } \\
\text { placas }\end{array}$ & $\begin{array}{l}\text { Todos los } \\
\text { equipos } \\
\text { electrificadoras } \\
\text { de alambrado } \\
\text { se alimentan de } \\
\text { una energía } \\
\text { convencional }\end{array}$ \\
\hline FOTO & 害章 & & 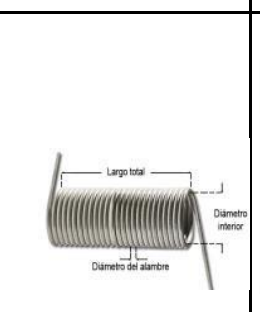 & & Uu UuU \\
\hline $\begin{array}{c}\text { VENTA } \\
\text { JAS }\end{array}$ & $\begin{array}{l}\text { Disminución } \\
\text { del volumen a } \\
\text { la presión } \\
\text { ejercida }\end{array}$ & $\begin{array}{l}\text { Ejerce su } \\
\text { acción hacia } \\
\text { el interior }\end{array}$ & $\begin{array}{l}\text { Los extremos } \\
\text { de los resortes } \\
\text { de torsión } \\
\text { pueden } \\
\text { doblarse }\end{array}$ & $\begin{array}{l}\text { En un } \\
\text { circuito se } \\
\text { comporta } \\
\text { como un } \\
\text { elemento } \\
\text { capaz de } \\
\text { almacenar } \\
\text { energía por } \\
\text { un periodo } \\
\text { de carga }\end{array}$ & $\begin{array}{l}\text { Económico, } \\
\text { rápida } \\
\text { colocación, } \\
\text { contención } \\
\text { eléctrica } \\
\text { independiente } \\
\text { de la } \\
\text { resistencia de } \\
\text { los materiales }\end{array}$ \\
\hline
\end{tabular}




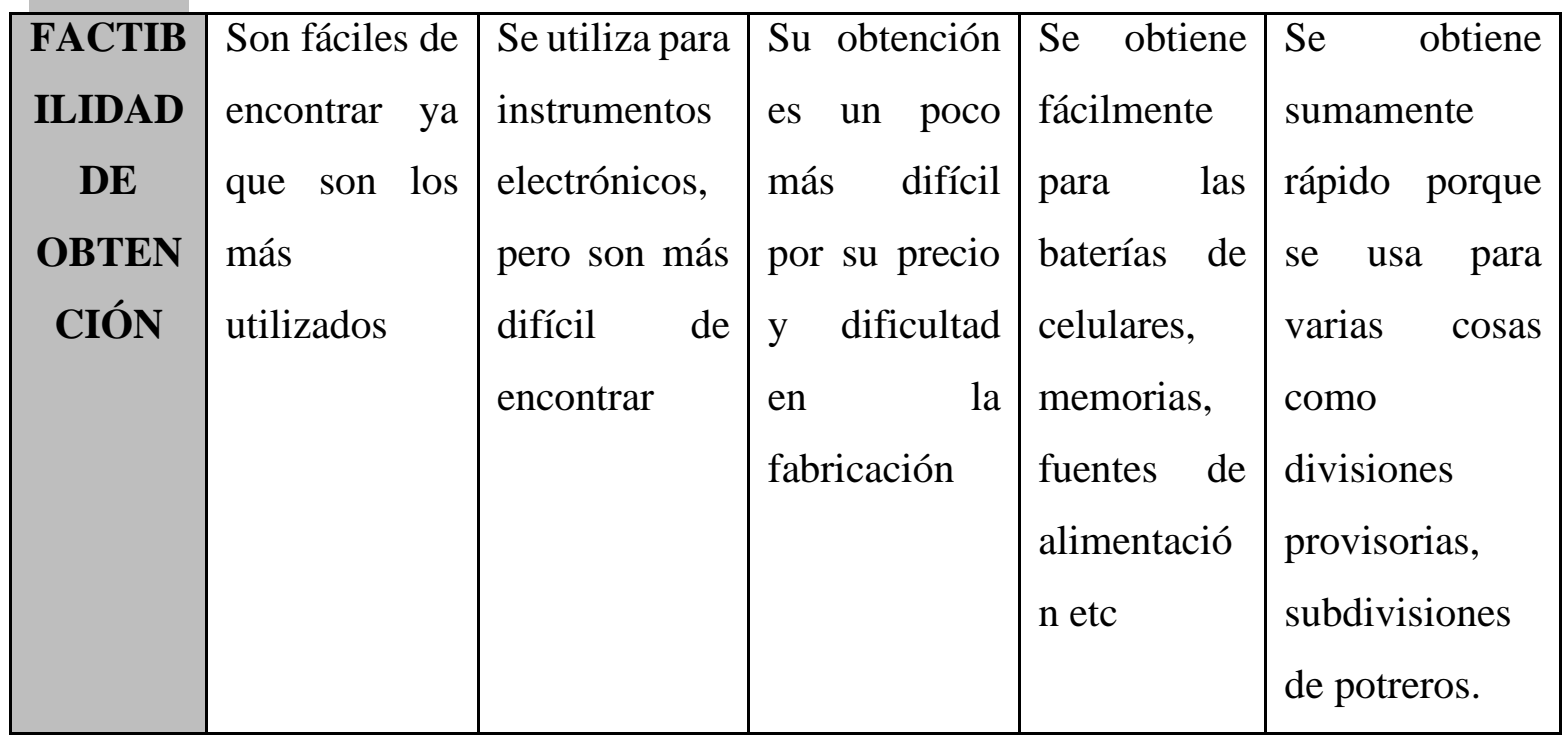

Tabla 2: Estudio del tipo y modelo del resorte, condensador y alambrado a utilizar.

\begin{tabular}{|c|c|c|}
\hline $\begin{array}{l}\text { PRODUCTO } \\
\end{array}$ & 1 & 2 \\
\hline $\begin{array}{l}\text { MATERIAL } \\
\text { CANALETA DE } \\
\text { SALIDA }\end{array}$ & Canaleta de jebe para piso & $\begin{array}{l}\text { Canaleta PVC } 20 \times 10 \mathrm{~mm} \\
\text { con Adhesivo }\end{array}$ \\
\hline PRECIO & $\mathrm{S} / 5.90$ & $\mathrm{~S} / 47.9$ \\
\hline CARACTERISTICA & $\begin{array}{l}\text { a) GRADO DE PROTECCION: IP45 } \\
\text { b) Material: PVC } \\
\text { c)AUTOEXTENGUIBLE }\end{array}$ & $\begin{array}{l}\text { : protege todo tipo de cables } \\
\text { que requieren pasar por el } \\
\text { piso. } \\
\text { a) GRADO DE } \\
\text { PROTECCION: IP46 }\end{array}$ \\
\hline IMAGEN & & \\
\hline
\end{tabular}




\begin{tabular}{|c|c|c|}
\hline VENTAJAS & $\begin{array}{l}\text { a) Auto extinguible: Material que } \\
\text { la fuente de ignición que le ha } \\
\text { combustionado deja de actuar sobre él. } \\
\text { b) Resistente a químicos de limpieza. } \\
\text { Con adhesivo. }\end{array}$ & $\begin{array}{l}\text { a) resistentes ante pisadas o } \\
\text { caída de cualquier objeto } \\
\text { sobre él. } \\
\text { b) Resistente a químicos de }\end{array}$ \\
\hline DEFICIENCIAS & $\begin{array}{l}\text { el adhesivo no es tan resistente, para el } \\
\text { corte de canaleta, debe ser muy exacto } \\
\text { y saber dónde ponerlo }\end{array}$ & $\begin{array}{l}\text { a) solo es para piso no tiene } \\
\text { para adaptación hacia la } \\
\text { pared o columna. }\end{array}$ \\
\hline $\begin{array}{l}\text { FACTIBILIDAD DE } \\
\text { OBTENCIÓN }\end{array}$ & $\begin{array}{l}\text { en cualquier supermercado de ventas } \\
\text { en construcción o también en las } \\
\text { ferreterías. }\end{array}$ & $\begin{array}{l}\text { en cualquier supermercado } \\
\text { de ventas en construcción o } \\
\text { también en las ferreterías. }\end{array}$ \\
\hline
\end{tabular}

Tabla 3: Estudio del tipo y modelo de canaleta.

\begin{tabular}{|l|l|l|}
\hline PRODUCTO & \multicolumn{1}{|c|}{1} & \multicolumn{1}{|c|}{2} \\
\hline MATERIAL & Tablero de triplay fenólico 9mm & $\begin{array}{l}\text { Plancha de Drywall Volcanita 1/2" } \\
\text { RF }\end{array}$ \\
\hline PRECIO & S/ 68.00 & S/ 30.50 \\
\hline CARACTERISTIC & $\begin{array}{l}\text { Es un panel derivado de la madera } \\
\text { con propiedades estructurales. } \\
\text { Mayor resistencia a la humedad. }\end{array}$ & $\begin{array}{l}\text { Las características de este sistema } \\
\text { constructivo permiten la utilización } \\
\text { en cualquier diseño, desde tabiques } \\
\text { divisorios y cielos rasos planos y } \\
\text { curvos hasta columnas cilíndricas, } \\
\text { rareas. } \\
\text { Material no conductor de } \\
\text { corriente. }\end{array}$ \\
\hline
\end{tabular}




\begin{tabular}{|c|c|c|}
\hline IMAGEN & & \\
\hline VENTAJAS & $\begin{array}{l}\text { Fácil de trabajar. El formato de } \\
\text { tablero facilita mucho el trabajo, y } \\
\text { al no usarse maderas } \\
\text { excesivamente densas también el } \\
\text { mecanizado. } \\
\text { Puede utilizarse en exteriores y/o } \\
\text { ambientes húmedos. Esta } \\
\text { característica viene condicionada } \\
\text { a la utilización de los adhesivos y } \\
\text { maderas adecuadas nara ello. } \\
\text { Facilidad para el curvado. }\end{array}$ & $\begin{array}{l}\text { Rápido: Gracias al corto tiempo de } \\
\text { instalación, los costos } \\
\text { administrativos y financieros se } \\
\text { reducen un } 35 \% \text { en comparación con } \\
\text { el sistema tradicional de } \\
\text { construcción. } \\
\text { Térmico: Le permite mantener cada } \\
\text { ambiente con su propia temperatura, } \\
\text { evitando pérdidas de energía en } \\
\text { lugares con aire acondicionado o } \\
\text { calefacción gracias a su } \\
\text { conductibilidad térmica de } 0.38 \\
\text { KCal/mh }{ }^{\circ} \mathrm{C} \text {. }\end{array}$ \\
\hline DEFICIENCIAS & $\begin{array}{l}\text { Posibilidad de existencia de } \\
\text { puntos débiles y/o vacíos. La } \\
\text { Madera tiene defectos naturales, } \\
\text { como por ejemplo los nudos. En } \\
\text { estos puntos la chapa es más débil, } \\
\text { y si además coinciden varios } \\
\text { nudos se puede ver resentida la } \\
\text { resistencia del conjunto. } \\
\text { Altamente inflammable. }\end{array}$ & $\begin{array}{l}\text { Se debe evitar exponerla a humedad } \\
\text { excesiva o extremas temperaturas. } \\
\text { La plancha de yeso no se recomienda } \\
\text { donde la temperatura excederá los } \\
212^{\circ} \mathrm{F}\left(52^{\circ} \mathrm{C}\right) \text { durante prolongados } \\
\text { períodos de tiempo. }\end{array}$ \\
\hline $\begin{array}{l}\text { FACTIBILIDAD } \\
\text { DE OBTENCION }\end{array}$ & $\begin{array}{lcc}\text { En cualquier supermercado } & \text { de } \\
\text { construcción } \quad \text { también } & \text { en } \\
\text { ferreterías y madereras. } & \end{array}$ & $\begin{array}{l}\text { En madereras y supermercado de } \\
\text { construcción }\end{array}$ \\
\hline
\end{tabular}


Tabla 4: Estudio y modelo del material base del sistema.

\begin{tabular}{|c|c|c|c|c|c|c|}
\hline Producto & 1 & 2 & 3 & 4 & 5 & 6 \\
\hline $\begin{array}{l}\text { Material } \\
\text { Piezoeléctrico }\end{array}$ & PZT-5A & PZT-5H & PMN-PZT & PMN-PT & AZO & PVDF \\
\hline PRECIO & $\begin{array}{l}10 \text { a } 100 \\
\text { dólares la } \\
\text { decena }\end{array}$ & $\begin{array}{l}\text { 30-200 } \\
\text { dólares } \\
\text { por ciento }\end{array}$ & $\begin{array}{l}\text { 1250-2000 } \\
\text { dólares } \\
\text { por medio } \\
\text { ciento }\end{array}$ & $\begin{array}{l}10.5-20 \\
\text { dólares } \\
\text { por } \\
\text { ciento }\end{array}$ & $\begin{array}{l}300 \\
\text { dólares } \\
\text { por } \\
\text { unidad }\end{array}$ & $\begin{array}{l}0.1-50 \\
\text { dólares } \\
\text { por } \\
\text { unidad }\end{array}$ \\
\hline $\begin{array}{l}\text { CARACTERIS } \\
\text { TICA }\end{array}$ & $\begin{array}{l}\text { Circular } \\
\text { de } \\
\text { Nitruro } \\
\text { de } \\
\text { aluminio }\end{array}$ & $\begin{array}{l}\text { Forma de } \\
\text { anillo }\end{array}$ & $\begin{array}{l}\text { Variabilid } \\
\text { ad en } \\
\text { modelos }\end{array}$ & $\begin{array}{l}\text { Cilindro } \\
\text { Negro }\end{array}$ & $\begin{array}{l}\text { Zirconio } \\
\text { y Titanio }\end{array}$ & En lámina \\
\hline Imagen & & & & & & \\
\hline Ventajas & $\begin{array}{l}\text { Más } \\
\text { amplio en } \\
\text { área. }\end{array}$ & $\begin{array}{l}\text { Agujero } \\
\text { para } \\
\text { mayor } \\
\text { agarre a } \\
\text { lo hare } \\
\text { Radio de } \\
15 \mathrm{~mm}\end{array}$ & $\begin{array}{l}\text { Mayor } \\
\text { masa de } \\
\text { cerámica } \\
\text { con ello } \\
\text { marmar } \\
\text { dofnrmani } \\
\end{array}$ & $\begin{array}{l}\text { Mayor } \\
\text { espesor }\end{array}$ & $\begin{array}{l}\text { Mayor }{ }_{\text {are }} \\
\text { durabili } \\
\text { dad por } \\
\text { flexión y } \\
\text { amon } \\
\text { Radio } \\
225 \mathrm{~mm}\end{array}$ & $\begin{array}{l}\text { Más } \\
\text { delgado y } \\
\text { de diseño } \\
\text { adecuado. } \\
\text { Diámetro } \\
20 \mathrm{~mm}, \\
21 \mathrm{~mm}, \\
25 \mathrm{~mm},\end{array}$ \\
\hline
\end{tabular}




\begin{tabular}{|c|c|c|c|c|c|c|}
\hline & & & & & & $\begin{array}{l}27 \mathrm{~mm}, \\
30 \mathrm{~mm}, \\
32 \mathrm{~mm}\end{array}$ \\
\hline Deficiencias & $\begin{array}{l}\text { El tipo de } \\
\text { conexión, } \\
\text { es } \\
\text { diferente } \\
\text { a } \quad \text { lo } \\
\text { propuesto }\end{array}$ & $\begin{array}{l}\text { Menor } \\
\text { área de } \\
\text { deformac } \\
\text { ión }\end{array}$ & $\begin{array}{l}\text { El tipo de } \\
\text { conexión, } \\
\text { no se } \\
\text { precisa los } \\
\text { polos }\end{array}$ & $\begin{array}{l}\text { Muy } \\
\text { pequeño } \\
\text { y de } \\
\text { salidas } \\
\text { de difícil } \\
\text { conexión }\end{array}$ & $\begin{array}{l}\text { El tipo } \\
\text { de } \\
\text { conexión } \\
\text {, no se } \\
\text { precisa } \\
\text { los polos }\end{array}$ & $\begin{array}{l}\text { Poca } \\
\text { durabilida } \\
\text { d y área de } \\
\text { contacto } \\
\text { menor }\end{array}$ \\
\hline
\end{tabular}

Tabla 5: Estudio y modelo de materiales Piezoeléctricos.

\section{Conclusiones:}

- La zona más transitada y con mayor movimiento ha de generar una cantidad mayor de energía, en la Universidad Nacional Tecnológica de Lima Sur es en la biblioteca Santiaga Antúnez de Mayolo.

- Se depende de las propiedades elásticas de las capas superficiales de las baldosas para poder generar eficientemente una deformación mecánica sobre el piezoeléctrico que posterior termine eso en energía.

- Por la dispersión de área se conviene centralizar el cableado generado por las baldosas en canaletas falsas en poder de organizar de forma eficiente el cableado para la alimentación de equipos que permite el funcionamiento de la red.

\section{Limitaciones del proyecto:}

Por motivos coyunturales del Virus COV.19, la elaboración de un prototipo funcional para la contratación de hipótesis y corroboración de los objetivos no ha sido posible. 


\section{Referencias:}

- E-STEP: Diseño de un prototipo para generación energética mediante tecnología piezoeléctrica. Aplicación a escaleras, Javier Ibáñez García, Universidad Politécnica de Catalunya, Junio 2012-España.

- First Steps towards Piezoaction, Dr. Lutz Pickelmann, 2010-Alemania.

- Diseño de un Colector de Energía Piezoeléctrico (Energy Harvesting) Mediante Optimización Topológica que Maximice la Transformación de Energía Mecánica en Eléctrica Generada por un Ser Humano al Caminar, Universidad Nacional de Colombia, Esteban Sepúlveda Orozco, Medellín 2014-colombia.

- Castellanos, N. (2013). Evaluación Preliminar del uso del efecto Piezoeléctrico para Generación de Energía. Revista Inventum. ed. 15. pp. 35 - 39.

- Bischur, E., \& Schwesinger, N. (2011). Piezoelectric energy harvester under parquet floor (Vol. 7977). San Diego, California, United States. doi:10.1117/12.880419 [doi]

- Bischur, E., \& Schwesinger, N. (2012). Energy harvestingfrom floor using organic piezoelectric modules. Wuhan, China: 2012 Power Engineering and Automation Conference. doi:10.1109/PEAM.2012.6612556.

- Shinjiro, T. (2006). Fabrication of (SrxK0.5-xNa0.5-x)NbO3 Pizoelectric Ceramics and Effects of MnO Addition on Their Piezoelectric Properties. japon: Materials Science and Engineering, The National Defense Academy. Obtenido de https://doi.org/10.1143/JJAP.45.7449.

- Xiaofeng Li, V. S. (2014). Modelling piezoelectric energy harvesting potential in an educational building (Vol. 85). (Elsevier, Ed.) Sydney, Australia. Obtenido de https://doi.org/10.1016/j.enconman.2014.05.096.

BIOTECH \& ENGINEERING Untels. Ene - jun.1(1), 2021; ISSN: 2788-4295; 82 - 99 